\title{
Cardiac Asthma
}

\author{
Tatsuya Tomomatsu
}

Kobe Medical College, Internal Medicine, Division 1.

This report has dealt with the cardiopulmonary function of the patients with. cardiac dyspnea in attempt to confirm the appearance of bronchoconstriction in the seizure of cardiac asthma.

\section{Method}

The patient was led to the laboratory on the earliest date of admission before instituting the medical treatment and either the cardiac or pulmonary function test was undertaken successively at $3 \mathrm{p} . \mathrm{m}$. and at $10 \mathrm{p} . \mathrm{m}$.. During the period of time between 3 and 10 the patient has been kept comfortable in the supine position and instructed to take a sleep after 8 p. m.

The hemodynamic of the pulmonary circulation has been measured by means of the extracorporeal measurement using radioisotope (RISA).

For the pulmonary function test spirometer and body plethysmography were employed.

\section{Results and Comment}

As shown in Table 1, comparing the degree of dyspnea between in daytime and at night of 44 cardiac patients, 7 had suffered from dyspnea with wheezing, 10 complained of aggravated distress of breathing and the rest of them had no trouble with respiration. The patients with increased dyspnea of the first two groups have been. in more serious condition. Changes found in cardiac output and pulmonary blood volume were not necessarily remarkable, because of the considerable variation of their original value from the normal limit. Those who suffered from nocturnal dyspnea are mostly found in the patients with the left ventricular failure.

Pulmonary function test was made on 22 cardiacs twice in daytime and at night, as shown in Table 2. Of 22 patients 16 complained of increased dyspnea with. abnormal breathing sound, indicating the definitely reduced pulmonary function. Decreases in arterial oxygen saturation $\left(\mathrm{SaO}_{2}\right)$ and carbon dioxide tension $\left(\mathrm{Paco}_{2}\right)$ were also found. In 7 patients with aggravated dyspnea at night airway resistance was found to be increased by means of body plethysmography.

It is proved that in those having nocturnal dyspnea rise in the arterial blood pressure is associated with reduction in cardiac output, increase in pulmonary blood volume and elevation in venous pressure. So far as the pulmonary function test is 
Table 1. Circulatory function in the cardiac patients measured in the daytime and at night

\begin{tabular}{|c|c|c|c|c|c|c|c|}
\hline \multicolumn{2}{|r|}{ Dyspnea } & wheezing & 7 cases & increased & 10 cases & none & 27 cases \\
\hline & Day & Night & Day & Night & Day & Night \\
\hline \multicolumn{2}{|r|}{$\mathrm{CR}$} & $93 \pm 17$ & $106 \pm 23$ & $82 \pm 13$ & $73 \pm 14$ & $71 \pm 5.7$ & $66 \pm 5.0$ \\
\hline \multicolumn{2}{|r|}{$\mathrm{RR}$} & $25 \pm 3.5$ & $29 \pm 4.1$ & $23 \pm 4.7$ & $23 \pm 5.4$ & $22 \pm 2.1$ & $21 \pm 2.0$ \\
\hline \multirow{2}{*}{$\stackrel{\rho_{1}}{m_{1}}$} & Syst. & $148 \pm 31$ & $158 \pm 31$ & $126 \pm 16$ & $132 \pm 19$ & $123 \pm 12$ & $114 \pm 8.5$ \\
\hline & Diast. & $82 \pm 35$ & $90 \pm 20$ & $72 \pm 28$ & $72 \pm 17$ & $80 \pm 15$ & $72 \pm 13$ \\
\hline \multicolumn{2}{|r|}{$\mathrm{VP}$} & $229 \pm 52$ & $315 \pm 39$ & $145 \pm 45$ & $156 \pm 34$ & $113 \pm 16$ & $125 \pm 17$ \\
\hline \multicolumn{2}{|r|}{ PCT } & $20 \pm 5$ & $24 \pm 7$ & $12 \pm 3$ & $14 \pm 4$ & $11 \pm 1$ & $13 \pm 2$ \\
\hline \multicolumn{2}{|r|}{$\mathrm{CI}$} & $2.4 \pm 0.7$ & $2.1 \pm 0.5$ & $3.0 \pm 0.3$ & $2.6 \pm 0.3$ & $3.2 \pm 0.4$ & $2.8 \pm 0.3$ \\
\hline \multicolumn{2}{|r|}{$\mathrm{SV} / \mathrm{M}^{2}$} & $24 \pm 5$ & $21 \pm 5$ & $57 \pm 16$ & $50 \pm 12$ & $26 \pm 24$ & $44 \pm 16$ \\
\hline \multicolumn{2}{|r|}{$\mathrm{PBV} / \mathrm{M}^{2}$} & $682 \pm 109$ & $729 \pm 163$ & $607 \pm 103$ & $642 \pm 142$ & $549 \pm 63$ & $589 \pm 74$ \\
\hline
\end{tabular}

Table 2. Pulmonary function in the cardiac patients measured in the daytime and at night

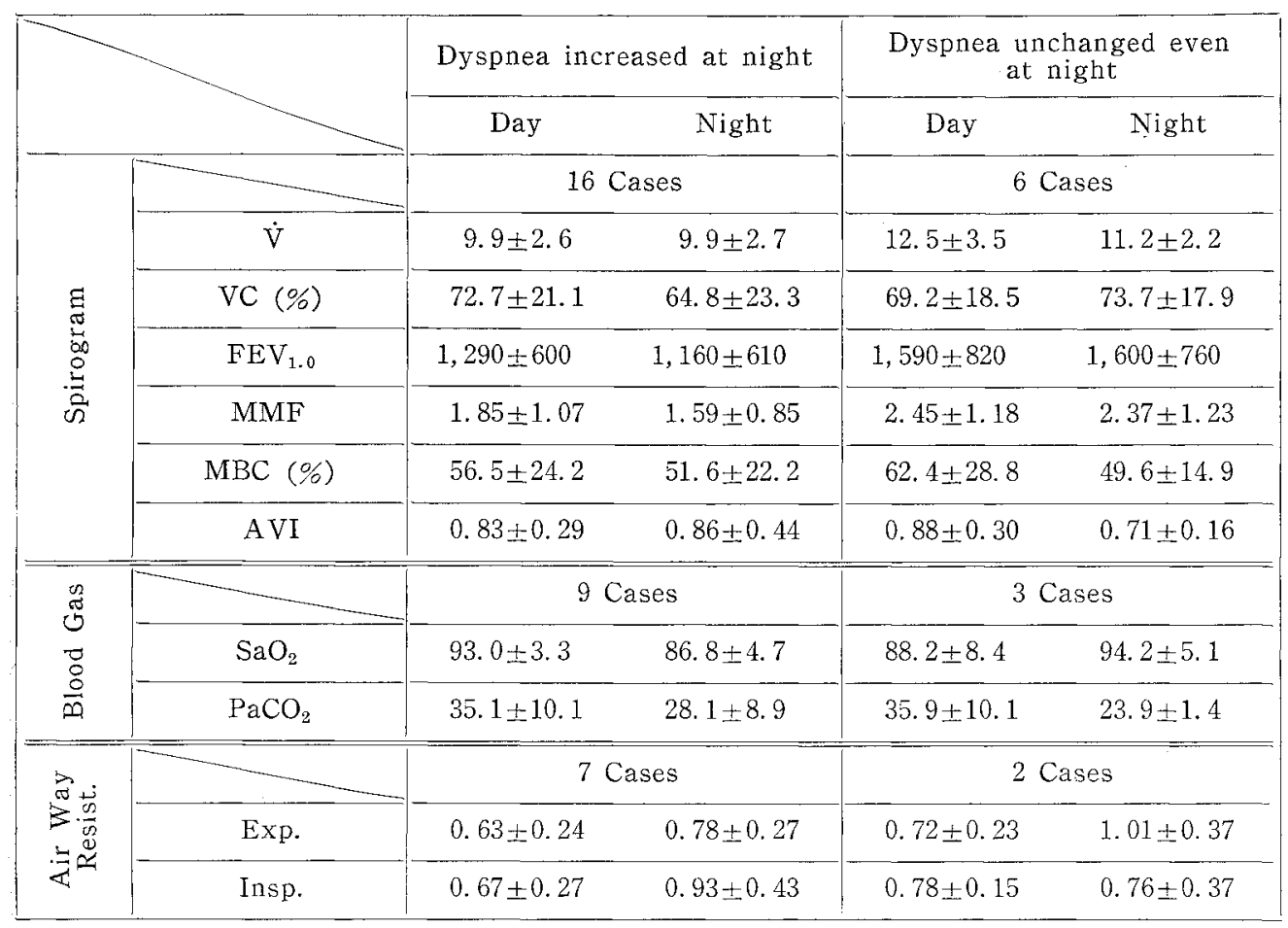


concerned, airway resistance is increased and followed by hyperventilation with the resultant hypoxemia and hypocapnia.

From foregoing data it is suggested that rise in the arterial blood pressure would be a kind of precipitating factor of the paroxysmal dyspnea. Attempts have been made to investigate whether the experimentally induced high blood pressure would evoke the dyspnea with wheezing in cardiac patients.

A) A simple infusion of $500 \mathrm{cc}$. to $1,000 \mathrm{cc}$. of $5 \%$ glucose before retiring cannot induce any respiratory distress.

B) Angiotensin $(30 \% . / 100 \mathrm{cc}$.) was given intravenously to 14 cardiacs, resulting in dyspnea with wheezing in 3 and without in 4 . Hemodynamic conditions in those 7 were more severe even before angiotensin loading than those in patients without dyspnea provoked.

Compared with the results (Table 3) obtained with epinephrine infusion which caused no detectable change in respiration in any subject studied, angiotensin brought about reduction in cardiac output, prolonged pulmonary circulation time and increased pulmonary blood volume preceded by rise of the arterial blood pressure, in spite of the fact that epinephrine injection was followed by increase in cardiac output and shortened pulmonary circulation time with almost identical increase in the arterial blood pressure.

Table 3. Comparative illustration of variations in circulatory measurements between angiotensin and epinephrine

\begin{tabular}{|c|c|c|c|c|c|}
\hline & \multirow[b]{2}{*}{ Dyspnea } & \multicolumn{2}{|c|}{ angiotensin } & \multicolumn{2}{|c|}{ epinephrine } \\
\hline & & \multicolumn{2}{|c|}{$(+) 7$ Cases } & \multicolumn{2}{|c|}{$(-) 5$ Cases } \\
\hline & & Before & After & Before & After \\
\hline & CR & $93.5 \pm 7.5$ & $115 \pm 15.9$ & $87.2 \pm 10.3$ & $108 \pm 19.3$ \\
\hline & $\mathrm{RR}$ & $23.0 \pm 3.6$ & $27.0 \pm 4.1$ & $21.0 \pm 3.2$ & $23.8 \pm 3.6$ \\
\hline \multirow{2}{*}{$\begin{array}{l}\dot{p u}^{\dot{ }} \\
\dot{\varphi}\end{array}$} & Syst. & $129 \pm 20.0$ & $160 \pm 15.5$ & $122 \pm 14.8$ & $172 \pm 21.2$ \\
\hline & Diast. & $79 \pm 23.5$ & $100 \pm 23.1$ & $80 \pm 18.3$ & $92 \pm 14.8$ \\
\hline \multicolumn{2}{|r|}{ VP } & $130 \pm 60.0$ & $182 \pm 65.0$ & $173 \pm 35.4$ & $211 \pm 33.9$ \\
\hline \multicolumn{2}{|r|}{ PCT } & $11.8 \pm 6.1$ & $17.8 \pm 7.4$ & $13.5 \pm 4.5$ & $9.1 \pm 3.3$ \\
\hline \multicolumn{2}{|r|}{$\mathrm{CI}$} & $2.77 \pm 0.89$ & $2.29 \pm 0.76$ & $2.87 \pm 0.26$ & $4.12 \pm 0.64$ \\
\hline \multicolumn{2}{|r|}{$\mathrm{SV} / \mathrm{M}^{2}$} & $29.2 \pm 36.3$ & $20.7 \pm 28.4$ & $33.5 \pm 6.0$ & $38.9 \pm 4.8$ \\
\hline \multicolumn{2}{|r|}{$\mathrm{PBV} / \mathrm{M}^{2}$} & $558 \pm 150$ & $634 \pm 166$ & $614 \pm 150$ & $603 \pm 192$ \\
\hline
\end{tabular}

C) Concerning the pulmonary function test in 5 of 10 patients with hypertension and in 2 of 7 with mitral valvular disease dyspnea with abnormal breathing sound was provoked with angiotensin. 
Rise in the arterial blood pressure was found to be remarkable in the hypertensive group, while it was slight and slow in the mitral group where in two cases the examination was forced to discontinue, because the severe breathing distress associated with wheezing resulted. In the patients with dyspnea provoked reduction in VC, FEV 1.0" and MMF and increased airway resistance were found. However, dyspnea was not induced with epinephrine.

It is revealed by the pulmonary function test that a type of dyspnea provoked with angiotensin is characterized by impaired passage through the airway.

Increase in the venous return has been postulated as a pathogenetic mechanism but it seems unlikely that the venous return is of prime importance in occurrence of the cardiac asthma, since the asthmatic breathing in cardiacs may develop even several hours after assuming the supine position. Furthermore, it has been provoked that onset of the asthma breathing may be suppressed in some cardiac patients with epinephrine which can produce pulmonary edema due in part to the rapid increase of the venous return. Since dyspnea cannot be provoked by the infusion of glucose to the cardiac patients in recumbency, even if venous return is kept greater while assuming the supine position, one should seek some other factor precipitating or contributory to the cardiac asthma. Rise of the blood pressure would be acting as a precipitating factor. The high blood pressure imposes an increased outflow load against the left ventricle, aggravating the cardiac failure and giving rise to an increase in the pulmonary blood volume.

Acceleration of the respiration results and the bronchospasm is elicited when asthmatic seizure occurs.

As an inherent asthma could be found in only 5 of 19 patients with cardiac asthma, some other unknown mechanism may have operated for facilitating the bronchial narrowing.

In the patients with nocturnal dyspnea both epinephrine and norepinephrine are found to be increased in early morning hour fraction. The pattern of urinary catecholamine was seen in the patients under the seizure of bronchial asthma.

Catecholamine in the early morning hour fraction restored to the normal limit, when the cardiac failure was subsided. It would be worthy of note whether catecholamine acts as a promotor or inhibitor to the cardiac asthma, but it is probable that catecholamine exerts an preventive effect on it, because epinephrine infusion cannot aggravate dyspnea.

\section{Conclusion}

1) The paroxysmal nocturnal dyspnea of cardiac origin is often preceded by rise in the arterial blood pressure and caused by the resultant left heart failure followed by the bronchial obstruction.

2) Increased urinary excretion of catecholamine, especially in early morning hour fraction is the common occurrence in the cardiac asthma, and exerts the possible beneficial influence on the critical condition. 